\title{
Comparison of IPSCs Evoked by Spiny and Fast-Spiking Neurons in the Neostriatum
}

\author{
Tibor Koos, ${ }^{1}$ James M. Tepper, ${ }^{2}$ and Charles J. Wilson ${ }^{3}$ \\ ${ }^{1}$ Section of Neurobiology, Yale University School of Medicine, New Haven, Connecticut 06510, ${ }^{2}$ Center for Molecular and Behavioral Neuroscience, Rutgers, \\ The State University of New Jersey, Newark, New Jersey 07102, and ${ }^{3}$ Department of Biology, University of Texas at San Antonio, San Antonio, Texas 78249
}

\begin{abstract}
Most neurons in the neostriatum are GABAergic spiny projection neurons with extensive local axon collaterals innervating principally other spiny projection neurons. The other source of GABAergic inputs to spiny neurons derives from a small number of interneurons, of which the best characterized are the parvalbumin-containing, fast-spiking interneurons. Spiny neuron collateral inhibition was not demonstrated until recently, because the IPSPs recorded at the soma are surprisingly small. In contrast, interneuronal inhibition was readily detected, comprising much larger IPSPs. Here, we report the application of quantal analysis and compartmental modeling to compare and contrast IPSCs in spiny neurons originating from axon collaterals and interneurons. The results indicate that individual release sites at spiny and interneuron synapses have similar quantal sizes and baseline release probabilities. Interneuronal unitary IPSCs are several times larger because of their proximal location on the neuron and because they have a larger number of transmitter release sites. Despite the small amount of current they can deliver to the soma, spiny cell collateral synapses had moderately high baseline release probabilities (0.5-0.9), suggesting that they are not weak because of some form of depression or modulation. The size of unitary collateral synaptic currents increased monotonically during development. These results argue against models of competitive inhibition in neostriatum, including those in which competitive inhibition is transiently effective during development and learning, and suggest a different role for the spiny cell axon collaterals.
\end{abstract}

Key words: IPSC; basal ganglia; interneuron; quantal analysis; development; GABA; inhibition

\section{Introduction}

Like the nearby forebrain regions of the cerebral cortex and hippocampus, the neostriatum contains fast-spiking, parvalbumincontaining (FS) interneurons that subserve feedforward inhibition of the principal cells. Unlike those other regions, however, the principal cells (the spiny cells) of the neostriatum do not contribute a recurrent synaptic excitation to the local circuit, but are GABAergic and inhibitory, both on each other and on the fast-spiking interneurons. This difference between the neostriatal network and that of the cortex has been the basis for speculations concerning its overall function (Wickens et al., 1995; Beiser and Houk, 1998; Plenz and Kitai, 2000; Bar-Gad and Bergman, 2001; Plenz, 2003). FS cells are relatively few in the neostriatum, but each contacts many spiny (Sp) cells within range of its 200- to 500$\mu \mathrm{m}$-diameter axonal arborization (Bolam et al., 1983; Kawaguchi, 1993; Kita, 1993; Kawaguchi et al., 1995). They exert a powerful inhibition on the Sp cells; a single action potential in one FS interneuron can veto spiking in a group of nearby $\mathrm{Sp}$ cells (Koos and Tepper, 1999). In contrast, the recurrent inhibition among

Received June 3, 2004; revised July 1, 2004; accepted July 3, 2004.

This work was supported by National Institutes of Health Grants NS20743 (C.J.W.), NS34865 (J.M.T.), and MH44866/MH38546 (Dr. Patricia S. Goldman-Rakic), and by a Busch Biomedical Support Grant (J.M.T.). We acknowledge the generous support and wise advice of Dr. Patricia Goldman-Rakic, in whose laboratory a portion of this work was performed.

Correspondence should be addressed to Dr. Charles J. Wilson, Department of Biology, University of Texas at San Antonio, 6900 North Loop 1604 West, San Antonio, TX 78249. E-mail: cjwilson@utsa.edu.

DOI:10.1523/JNEUROSCI.2163-04.2004

Copyright $\odot 2004$ Society for Neuroscience $\quad$ 0270-6474/04/247916-07\$15.00/0 the spiny cells is so weak that studies using sharp electrodes failed to observe it at all (Jaeger et al., 1994), unless hundreds of trials were averaged to reveal IPSPs only a fraction of a millivolt in amplitude (Tunstall et al., 2002). Subsequently, whole-cell recording studies, most using chloride loading to increase the driving force, have revealed that the synapses among spiny cells may be very weak but are very common (Czubayko and Plenz, 2002; Guzman et al., 2003; Taverna et al., 2004).

The reason these plentiful synapses are not more effective is still uncertain, as is the significance of combining a divergent powerful feedforward inhibition and a convergent weak inhibitory feedback on approximately the same spatial scale. On theoretical grounds, it has been suggested that collateral inhibition may be important only during periods of adjustment of synaptic strength [e.g., during learning or synaptogenesis (Foldiak, 1990; Plenz and Kitai, 2000; Bar-Gad and Bergman, 2001)], and may be effectively turned off at other times. Thus, the connections among spiny neurons may, at the time of the experiment, be in a state of reduced synaptic strength because of reduced postsynaptic sensitivity or release probability. If so, studies of collateral inhibition in slices taken from adult animals under laboratory conditions might underestimate the strength of this connection.

Here, we compare the strength of unitary synaptic conductances in principal cells evoked by a nearby spiny cell or FS interneuron, including the size of the quantal conductance change, the baseline (low repetition rate) probability of release, and the time course of the synaptic conductance at the two synapses. We also 
estimate the effect of differential location of the two synapse types on the surface of the spiny cell.

\section{Materials and Methods}

Neostriatal slices were obtained from Sprague Dawley rats of both sexes. Surgical procedures were performed in accordance with the National Institutes of Health Guide to the Care and Use of Laboratory Animals and with the approval of university animal care and use committees at Rutgers University, the University of Texas, and Yale University. Animals were deeply anesthetized with a mixture of ketamine and xylazine. Subsequently, animals were transcardially perfused with partially frozen perfusion solution containing the following (in $\mathrm{mm}$ ): 225.0 sucrose, $2.5 \mathrm{KCl}$, $0.5 \mathrm{CaCl}_{2}, 7.0 \mathrm{MgCl}_{2}, 28.0 \mathrm{NaHCO}_{3}, 1.25 \mathrm{NaH}_{2} \mathrm{PO}_{4}, 7.0$ glucose, 1.0 ascorbate, and 3.0 pyruvate, and saturated with $95 \% \mathrm{O}_{2}-5 \% \mathrm{CO}_{2}$. Perfusion of $60-150 \mathrm{ml}$ of solution was performed at high rates $(50-200$ $\mathrm{ml} / \mathrm{min}$ ), and slices were cut in the same solution to a thickness of 300 or $400 \mu \mathrm{m}$ in an oblique-to-horizontal plane parallel to the corticostriatal fiber bundles. Slices were stored at $35^{\circ} \mathrm{C}$ in normal Ringer's solution for $\sim 20 \mathrm{~min}$ and subsequently cooled to room temperature (RT). The normal Ringer's solution contained the following (in $\mathrm{mm}$ ): $125.0 \mathrm{NaCl}, 2.0$ $\mathrm{KCl}, 2.0 \mathrm{CaCl}_{2}, 1.0 \mathrm{MgCl}_{2}, 25.0 \mathrm{NaHCO}_{3}, 1.25 \mathrm{NaH}_{2} \mathrm{PO}_{4}$, and 25.0 glucose. Recordings were obtained at RT or at $32^{\circ} \mathrm{C}$.

Whole-cell recordings in slices from adults were obtained with $1.5 \mathrm{~mm}$ borosilicate pipettes exhibiting 5-8 (presynaptic) or 2.5-4 (postsynaptic) $M \Omega$ resistance. Presynaptic neurons were recorded with an intracellular solution containing the following: $120.0 \mathrm{~mm} \mathrm{~K}$ gluconate, $20.0 \mathrm{~mm}$ $\mathrm{KCl}, 0.02$ mM EGTA, 10.0 mM HEPES, $2.0 \mathrm{~mm} \mathrm{MgCl}_{2}, 4.0$ mм Na $\mathrm{N}_{2} \mathrm{ATP}$, $0.4 \mathrm{mM} \mathrm{Na}_{2} \mathrm{GTP}$, and $0.3-0.5 \%$ biocytin. Postsynaptic neurons were voltage clamped at $-70 \mathrm{mV}$ with pipettes containing the following: 140.0 $\mathrm{mm} \mathrm{CsCl}, 10.0 \mathrm{~mm}$ EGTA, $10.0 \mathrm{~mm}$ HEPES, $2.0 \mathrm{~mm} \mathrm{MgCl}_{2}, 4.0 \mathrm{~mm}$ $\mathrm{Na}_{2}$ ATP , $0.4 \mathrm{~mm} \mathrm{Na}_{2} \mathrm{GTP}, 5.0 \mathrm{~mm}$ lidocaine $N$-ethyl bromide (QX-314), and $0.25-0.4 \%$ biocytin; $E_{\mathrm{Cl}-}=2 \mathrm{mV}$. In 10 adult pairs, $40.0 \mathrm{~mm} \mathrm{~K}$ gluconate was added, and the concentrations of CsCl, EGTA, and QX314 were reduced to $96.0,5.0$, and $2.0 \mathrm{~mm}$, respectively, resulting in $E_{\mathrm{Cl}-}$ $=-7.3 \mathrm{mV}$. Paired recordings were made with an Axon Instruments (Foster City, CA) Multiclamp 700A amplifier, a Neurodata Instruments (New York, NY) IR-283, or an EPC-9 (HEKA Elektronik, Lambrecht/ Pfalz, Germany) amplifier. Fast and slow capacitance compensations were used. Access resistance was periodically monitored using the slow capacitance compensation circuit and was maintained at a low value when necessary by applying small pressure pulses to the recording pipette. Voltage-clamp recordings were not analyzed quantitatively if the uncompensated access resistance exceeded $30 \mathrm{M} \Omega$. Series resistance $\left(R_{\mathrm{s}}\right)$ values were typically $12-18 \mathrm{M} \Omega$. In some cases, series resistance was compensated for by $<50 \%$ with a correction circuit. Presynaptic and postsynaptic recordings were digitized at $10-20 \mathrm{kHz}$. Postsynaptic voltage-clamp recordings were filtered at $2-5 \mathrm{kHz}$ on-line.

Connected pairs were found by first obtaining a recording from a neuron, and then conducting a serial search for a connected presynaptic neuron by stimulating neurons in the vicinity in cell-attached mode. Connected neurons were then recorded in the whole-cell mode with a clean pipette. Only those pairs for which the final whole-cell recording was successful and allowed verification of the identity of the presynaptic neuron were considered. All of the reported data refer to IPSCs that were elicited by stimulating the presynaptic neuron in whole-cell mode, with the exception of demonstrating the existence of slow depression of the IPSC in cell-attached mode. In these latter pairs, the presynaptic neurons were subsequently verified to be MS neurons with whole-cell recording.

Slices taken from developing animals were prepared as described above, but recordings were performed using electrodes filled with the following (in mM): $130.5 \mathrm{~K}$ methylsulfate, $10.0 \mathrm{KCl}, 7.5 \mathrm{NaCl}, 10 \mathrm{HEPES}$, 0.2 EGTA, $2 \mathrm{MgATP}, 0.2 \mathrm{MgGTP}$, and 0.01 phosphocreatine $\left(E_{\mathrm{Cl}-}=\right.$ $-52 \mathrm{mV}$ ). In approximately one-half of the experiments, perforatedpatch recordings were performed using electrodes containing $100 \mu \mathrm{M}$ gramicidin (Akaike, 1997), to avoid perturbing the chloride equilibrium potential. Tests for synaptic connections were made in both directions, using an Axopatch 200B amplifier. The postsynaptic neuron in each test was held at $-30 \mathrm{mV}$, and the presynaptic neuron was stimulated to fire
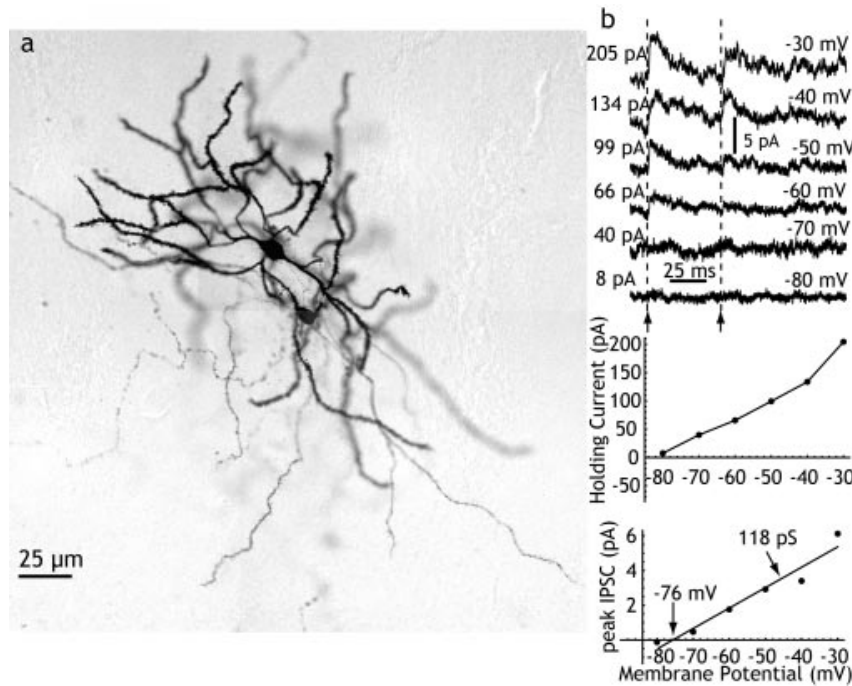

Figure 1. Synaptic connections between striatal spiny neurons. $a$, Pair of synaptically connected striatal spiny cells stained with biocytin and located $\sim 50 \mu \mathrm{m}$ apart after paired wholecell recording in an adult rat. $b, S p \rightarrow S p$ IPSC recorded in perforated-patch configuration in a 13-d-old rat. Pairs of action potentials were evoked in the presynaptic spiny neuron at the arrows, at various postsynaptic holding potentials as indicated at the right of each trace. The current required to hold the soma at the various potentials is shown in the graph at the top, and the amplitude of the IPSC is likewise shown below that. The IPSC reversed at $-76 \mathrm{mV}$, and the slope of the I-V relation (the apparent conductance change at the soma) was only $118 \mathrm{pS}$. IPSCS were typically $<5 \mathrm{pA}$ in amplitude, even at $-30 \mathrm{mV}$ (driving force, $\sim 40 \mathrm{mV}$ ).

using pairs of current pulses. Series resistances in the postsynaptic neuron were $20 \mathrm{M} \Omega$ or less.

Data analysis was performed using custom-made software written in IgorPro (WaveMetrics, Lake Oswego, OR) and in Mathematica (Wolfram Research, Champaign, IL). Sample values are reported as mean \pm SD.

\section{Results}

\section{Unitary IPSCs evoked by spiny cells}

Pairs of spiny neurons were studied in slices obtained from adult animals (131-310 gm; $n=39$ ) and from immature animals (6-18 d; $n=43)$ to determine whether the strength of these synaptic contacts were particularly effective at some time during development (e.g., during corticostriatal synaptogenesis). Spiny neurons were identified by their differential interference contrast (DIC) microscopic appearance and characteristic firing patterns during recording, and also by biocytin staining (Fig. 1). For the developmental comparison, the chloride reversal potential was not purposely disturbed, and many of these pairs were recorded using perforated-patch recording with gramicidin to prevent any disturbance of the intracellular chloride concentration. These experiments confirmed the presence of very weak synaptic connections among many immature striatal spiny neurons. Pairs of spiny neurons from animals younger than $11 \mathrm{~d}(n=22)$ never yielded synaptic connections, in agreement with previous studies showing that the axon collaterals of spiny cells become mature at approximately that time (Tepper et al., 1998). Between postnatal days 11 and 14, the probability of a synaptic connection between two spiny neurons located within $100 \mu \mathrm{m}$ of each other was one-sixth (2 of 12), and adult-like connectivity was seen starting at approximately day 15, with three of nine pairs being connected. Because the test for synaptic connections was bidirectional, and we did not observe any reciprocally connected pairs of spiny neurons, this translates to a one-sixth probability of connections in either direction, which is in agreement with our work in adult animals and previous reports (Czubayko and Plenz, 
2002; Tunstall et al., 2002; Guzman et al., 2003; Taverna et al., 2004). The synaptic currents evoked in all of the pairs in which the chloride equilibrium potential was not disturbed was very small, often just barely above detection threshold when 10-50 of the IPSCs were averaged, and ranged from 2 to $6 \mathrm{pA}$ when holding the membrane potential at $-30 \mathrm{mV}$. The reversal potential of these synaptic currents was measured in two perforated-patch recordings with the highest IPSC amplitude and shortest rise times, and in both cases measured $-76 \mathrm{mV}$ (Fig. 1).

In adult animals, 39 connected pairs of spiny cells were studied using one electrode filled with a conventional whole-cell recording solution containing high $\mathrm{K}^{+}$and low $\mathrm{Cl}^{-}$, and the other electrode filled with a Cs ${ }^{+}$substitution of $\mathrm{K}^{+}$and high $\mathrm{Cl}^{-}(144$ $\mathrm{mM})$. This arrangement allowed detection of IPSCs in only one direction, and yielded a connection rate of $12 \%$ (39 of 325 pairs). The postsynaptic cell (recorded using the high $\mathrm{Cl}^{-}$and $\mathrm{Cs}^{+}$electrode) was held at $-70 \mathrm{mV}$, yielding a chloride driving force of $+68 \mathrm{mV}$. The input resistance of the postsynaptic cell was increased by $\mathrm{Cs}^{+}$replacement $($mean $=128 \pm 35 \mathrm{M} \Omega, n=11$, vs $30.3 \pm 5.6 \mathrm{M} \Omega$ in a sample of five control cells measured at -85 $\mathrm{mV})$. Cesium replacement also altered the resting membrane potential of spiny neurons (to $-21 \mathrm{mV}$ ), as indicated by the zerocurrent point in voltage clamp. Correspondingly, the amplitudes, rise times, and half-widths of the IPSCs evoked by neighboring spiny cells were greatly altered in the high $\mathrm{Cl}^{-}$, high $\mathrm{Cs}^{+}$recordings. For 26 connected pairs studied in detail, IPSC amplitudes ranged from 5 to $123 \mathrm{pA}(51 \pm 39 \mathrm{pA})$, rise times varied from 0.7 to $2.2 \mathrm{msec}(1.3 \pm 0.6 \mathrm{msec})$, and half-widths ranged from 5.7 to $15.3 \mathrm{msec}(12.9 \pm 3.4 \mathrm{msec})$. Latencies from the rising phase of the presynaptic spike varied from 1.7 to $3.3 \mathrm{msec}(2.4 \pm 0.51$ msec; $n=8$ ). The decaying phase of the IPSC was adequately fit by a single exponential decay with a time constant ranging from 6.7 to $17.1 \mathrm{msec}(12.4 \pm 9.5 \mathrm{msec})$. In three connected pairs recorded using high $\mathrm{Cl}^{-}$but without $\mathrm{Cs}^{+}$, IPSC amplitudes were smaller, ranging from 8.2 to $34.0 \mathrm{pA}(18.3 \pm 13.8 \mathrm{pA})$. These data are in close agreement with results of previous studies (Taverna et al., 2004), and further indicate that blockade of $\mathrm{K}^{+}$channels with intracellular $\mathrm{Cs}^{+}$increases the apparent amplitude of the $\mathrm{Sp} \rightarrow \mathrm{Sp}$ IPSC approximately threefold. The use of intracellular $\mathrm{Cs}^{+}$increases the estimate of mean synaptic conductance for the $\mathrm{Sp} \rightarrow \mathrm{Sp}$ IPSC in adult cells from 0.27 to $0.75 \mathrm{nS}$.

\section{Unitary IPSCs evoked by FS cells}

IPSCs evoked in spiny cells by FS neurons were studied in 10 $\mathrm{FS} \rightarrow$ Sp pairs in which the postsynaptic neuron was held at -70 $\mathrm{mV}$ and $\mathrm{Cl}^{-}$loaded to adjust its reversal potential to $\sim 0 \mathrm{mV}$. FS cells were initially identified by their appearance in DICvisualized slices, subsequently by their characteristic firing pattern when driven with depolarizing current pulses, and finally by their appearance when stained by intracellular application of biocytin (Koos and Tepper, 1999). The rise times of these IPSCs ranged from 0.6 to $10 \mathrm{msec}$. The pair with the longest rise time also had the smallest amplitude, suggesting that it was inadequately clamped, and it was thus removed from the sample, giving a range of $0.6-3 \mathrm{msec}(1.5 \pm 2.9 \mathrm{msec} ; n=9)$, with halfwidths ranging from 5.9 to $17.9 \mathrm{msec}(11.6 \pm 3.8 \mathrm{msec})$, consistent with electron microscopic observations indicating that
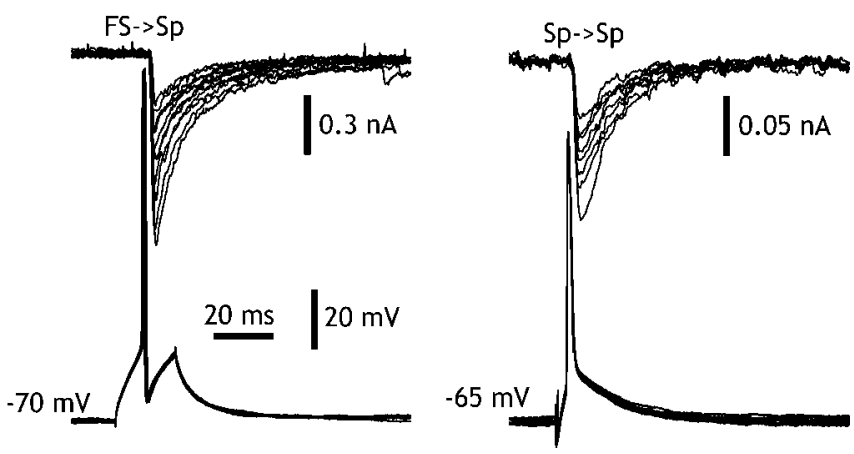

Figure 2. Comparison of IPSCs evoked in striatal spiny neurons by other spiny cells $(\mathrm{Sp} \rightarrow \mathrm{Sp}$ ) and by fast-spiking cells (FS $\rightarrow$ Sp). Typical IPSCs evoked by FS cells and by Sp cells in whole-cell recordings after replacement of $\mathrm{K}^{+}$by $\mathrm{Cs}^{+}$in the postsynaptic neuron to minimize electrotonic distance effects, and adjustment of the $\mathrm{Cl}^{-}$reversal potential to $\sim 0 \mathrm{mV}$. The time courses of the IPSCs are similar, but the FS-evoked IPSP is approximately six times larger than that evoked by the spiny neuron. Note the difference in scales.

these synapses are located on the somata and proximal dendrites of the spiny cells, and so their amplitudes and time courses would be accurately represented in voltage-clamp measurements. Averages of IPSCs evoked at a stimulation rate of $1 / \mathrm{sec}$ or less yielded average peak synaptic currents ranging from 45 to $570 \mathrm{pA}$ (269 \pm $213 \mathrm{pA} ; n=9$ ). Given a driving force of $70 \mathrm{mV}$, these yielded a mean peak conductance of $3.84 \mathrm{nS}$. Decay of the IPSCs was adequately represented by a single exponential process, with a time constant of $11.4 \pm 2.1 \mathrm{msec}$. These results were comparable with the corresponding values for $\mathrm{Sp} \rightarrow \mathrm{Sp}$ synapses, except that the amplitude was significantly larger $(t=3.07$; $\mathrm{df}=34 ; p<0.01)$ (Fig. 2).

\section{Quantal comparison of FS and spiny cell synapses}

The difference in sizes of synaptic currents evoked by FS and spiny neurons suggests that these synapses may differ in their postsynaptic response to single quanta, in the probability of quantal release, and/or in the number of release sites available for release (perhaps synapses formed) by the axon and the postsynaptic cell. To distinguish among these possibilities, we applied the variance-mean analysis (Clements and Silver, 2000) and nonstationary PSC fluctuation analysis (Scheuss et al., 2002), from which release probability $\left(p_{\mathrm{i}}\right)$, quantal current $\left(q^{*}\right)$, and the number of release sites $(N)$ can be extracted (Table 1$)$.

We performed this analysis on eight $\mathrm{Sp} \rightarrow \mathrm{Sp}$ pairs for which voltage clamp was considered adequate based on a short $(<2$ msec) IPSC rise time and an access resistance of $<10 \mathrm{M} \Omega$. An example is shown in Figure 3. Ten presynaptic action potentials were evoked at $20 \mathrm{msec}$ intervals. Mean and variance were calculated for IPSCs evoked in the postsynaptic spiny neuron by each action potential in the train. $\mathrm{Sp} \rightarrow \mathrm{Sp}$ IPSCs exhibited pronounced short-term depression, which was largest between the first and second presynaptic action potentials, and which recovered completely within $500 \mathrm{msec}$ (an 11th stimulus was applied to test recovery). This relationship was maintained across successive trials (Fig. 3b). There was often also a long-term depression across subsequent trials (Fig. $3 b$ ). The slower depression was not

Table 1. A comparison of peak unitary IPSC, rise time, and half-width of the unitary IPSC, and the quantal parameters for Sp $\rightarrow$ Sp pairs and FS $\rightarrow$ SP pairs

\begin{tabular}{llllll}
\hline Group & Peak current $(\mathrm{pA})$ & Rise time & Half-width (msec) & Number of release sites & $q^{*}(\mathrm{pA})$ \\
\hline $\mathrm{Sp} \rightarrow$ Sp & $51 \pm 39$ & $1.3 \pm 0.6$ & $12.9 \pm 3.4$ & $2.9 \pm 1.6$ & $43 \pm 16$ \\
$\mathrm{FS} \rightarrow$ Sp & $269 \pm 213$ & $1.6 \pm 1.0$ & $11.6 \pm 3.8$ & $6.7 \pm 7.8$ & $0.76 \pm 0.15$ \\
\hline
\end{tabular}



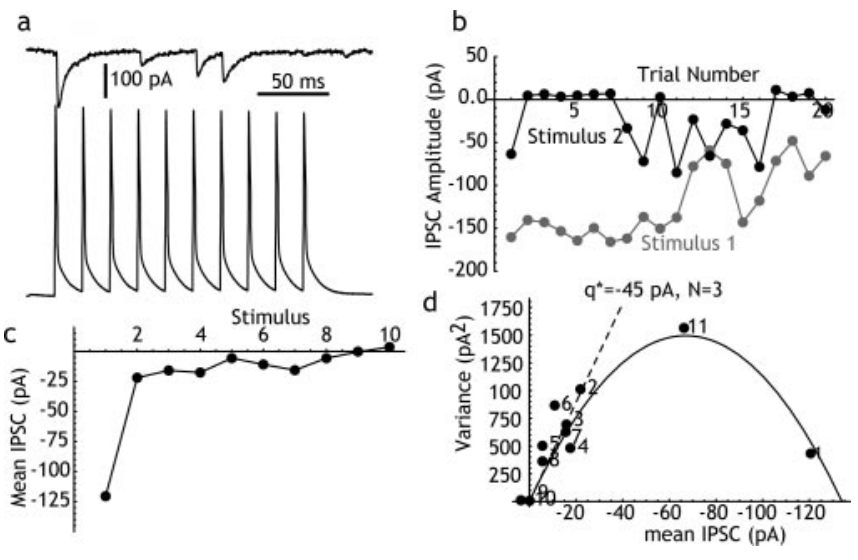

Figure 3. Mean-variance analysis of $S p \rightarrow S p$ IPSCs. $a$, A single trial showing the IPSCs evoked in a spiny neuron by repeated firing of a nearby connected spiny neuron at $20 \mathrm{msec}$ intervals. Note frequent failures. $b$, Long-term stability of the amplitude of the IPSC evoked over 20 repeated trials. The response to the first and second spikes in the sequence are plotted. There was a long-term decrease in the response to the first stimulus. To reduce the effects of such trends, variance was calculated using sequential responses, as described by Scheuss et al. (2002). c, Response to each spike in the train, averaged over trials. Short-term depression of the IPSC occurred primarily between the first and second trials. $d$, Mean-variance analysis of the IPSC across responses in the train. The points are labeled with the stimulus number. The point numbered 11 is an additional presynaptic spike evoked $500 \mathrm{msec}$ after the end of the stimulus train. The points describe a parabola, corresponding to a quantal IPSC $\left(q^{*}\right.$, from the initial slope of the parabola) of $45 \mathrm{pA}$ and three release sites. For this cell, the probability of release for the first stimulus was almost 0.9 .

associated with any change in the holding current or any other measure of the integrity of the neuron or the recording. To correct for its effects, variance was measured using sequential trials as described by Scheuss et al. (2002), and in some cells, it was also possible to perform the analysis on a subset of trials in which there was little or none of the slow depression (e.g., the first 10 trials in Fig. 3). These methods yielded similar values for $q^{*}$ and $N$, suggesting that the slow depression, like the short-term plasticity, was primarily caused by a reduction in release probability.

The mean-variance analysis in eight $\mathrm{Sp} \rightarrow \mathrm{Sp}$ pairs indicated that these synapses are characterized by a small number (one to six) of release sites $(2.9 \pm 1.6)$, with a quantal size between 0.019 and $0.063 \mathrm{nA}(0.043 \pm 0.016 \mathrm{nA})$, and a high initial (low stimulation rate) release probability $(0.76 \pm 0.15)$. Considering the 68 $\mathrm{mV}$ driving force for chloride in these experiments (calculated from intracellular and extracellular $\mathrm{Cl}^{-}$concentrations), the peak quantal conductance change at these synapses varied from 271 to $900 \mathrm{pS}$, corresponding to $\sim 8-27 \mathrm{GABA}_{\mathrm{A}}$ channels open, based on an open-channel conductance of 33 pS (Kilman et al., 2002).

One $\mathrm{FS} \rightarrow$ Sp pair was studied using the nonstationary IPSC fluctuation method as described above and three others by variation of transmitter-release probability using muscarine (Koos and Tepper, 2002). These two methods gave similar results, with $q^{\star}$ ranging from 0.050 to $0.085 \mathrm{nA}(0.064 \pm 0.017 \mathrm{nA})$, low rate probability of release ranging from 0.34 to $0.82(0.57 \pm 0.24)$, and for $N$ ranging from 1 to 18 (mean $=6.7 \pm 7.8)$. The results from the pair studied using repetitive stimulation are shown in Figure 4. These values for $q^{*}$ are similar to those seen for $S p \rightarrow S p$ pairs, indicating that the difference between the synapses formed by $\mathrm{FS} \rightarrow \mathrm{Sp}$ and $\mathrm{Sp} \rightarrow \mathrm{Sp}$ pairs (after removal of the effects of electrotonic placement by Cs replacement) may be in the number of release sites. Responses beyond the first two or three presynaptic spikes often fell below the parabola defined by early responses, perhaps because of desensitization (Scheuss et al., 2002).
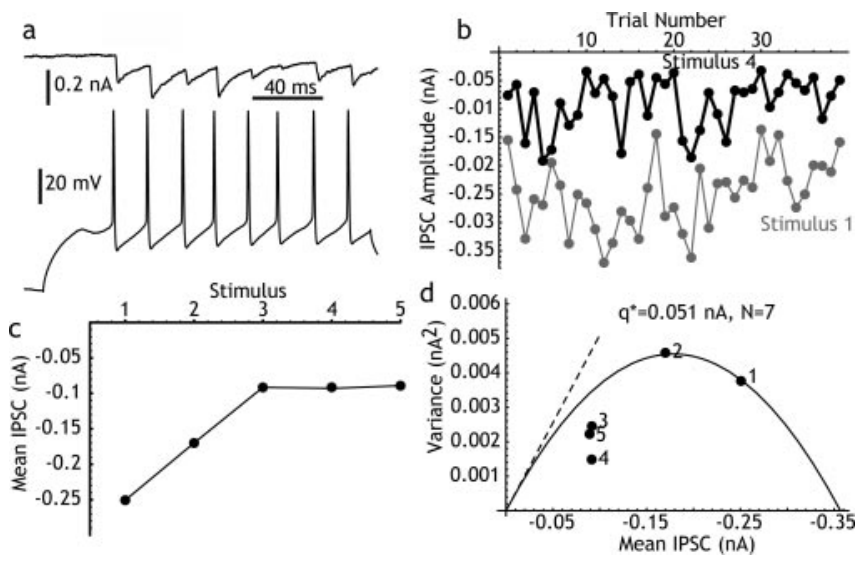

Figure 4. Mean-variance analysis of FS $\rightarrow$ Sp IPSCs. $a, A$ single trial showing the IPSCS evoked in a spiny neuron by repeated firing of a nearby connected FS neuron. Note absence of failures. $b$, Long-term stability of the response over 40 repeated trials, as in Figure 3, comparing the first and fourth IPSC. c, Short-term depression in the response, for the first five spikes in the train. Because firing in the FS cell was evoked by a constant current pulse, there was not always the same number of presynaptic action potentials on each trial, and the analysis was restricted to the first five spikes. $d$, Mean-variance analysis for this pair. The first two IPSCs describe a parabola with a quantal IPSC of $0.051 \mathrm{nA}$ and seven release sites. Probability of release on the first spike is 0.8 .

\section{Electrotonic structure of the spiny cell}

Current clamp results of others (Czubayko and Plenz, 2002; Tunstall et al., 2002; Guzman et al., 2003), when compared with our previous data (Koos and Tepper, 1999), suggest that the amplitude of $\mathrm{FS} \rightarrow \mathrm{Sp}$ synapses is several times larger than that of $\mathrm{Sp} \rightarrow \mathrm{Sp}$ synapses, measured at the soma. Our voltage-clamp results using high $\mathrm{Cs}^{+}$to reduce the electrotonic distance between the somatic point clamp and dendritic synapses yield a smaller difference in conductance change, approximately threefold. To determine whether the effects of replacing $\mathrm{K}^{+}$with $\mathrm{Cs}^{+}$on IPSC amplitude were consistent with the electrotonic structure of the spiny neuron, we constructed a computational model of the spiny neuron, using established morphological features of these cells (Wilson, 1992). The model was constructed using Neuron (Hines and Carnevale, 1997) and consisted of a 12- $\mu \mathrm{m}$-diameter soma; 6 primary dendrites, $10 \mu \mathrm{m}$ in length and tapering from 2.5 to $2.0 \mu \mathrm{m}$ in diameter; 12 secondary dendrites, $20 \mu \mathrm{m}$ in length and tapering from 2.0 to $1.0 \mu \mathrm{m}$ in diameter; and 24 tertiary dendrites, each $180 \mu \mathrm{m}$ in length and tapering from 0.96 to 0.3 $\mu \mathrm{m}$. The tertiary dendrites were spiny. Dendritic spines were represented by increasing both the effective membrane conductance and capacitance of the tertiary dendrite by a constant factor (SpA) that corresponded to the proportional increase of the dendritic surface area attributed to dendritic spines. The model was used to simulate our voltage-clamp data. Membrane conductivity $\left(g_{\mathrm{m}}\right)$ was assumed to be constant over the voltage ranges obtained (all of the variation was caused by voltage-clamp errors), and a single cytoplasmic resistivity $\left(R_{\mathrm{i}}\right)$ was used throughout the cell. These parameters were determined by voltage-clamp experiments in which the clamp current was measured using a small $(10 \mathrm{mV})$ hyperpolarizing voltage pulse with no correction of cell capacitance or access resistance, as shown in Figure $5 a$. These measurements were made in cells recorded with $\mathrm{Cs}^{+}{ }^{+} \mathrm{re}-$ placement. The parameter-fitting capability of Neuron was used to find best fits of $\mathrm{SpA}, g_{\mathrm{m}}$, and $R_{\mathrm{i}}$ [and also the series resistance $\left(R_{\mathrm{s}}\right)$ ] for the anatomically accurate spiny neuron, both under $\mathrm{Cs}^{+}$replacement (high input resistance) and control (low input resistance) experimental conditions. At holding values of 60-70 


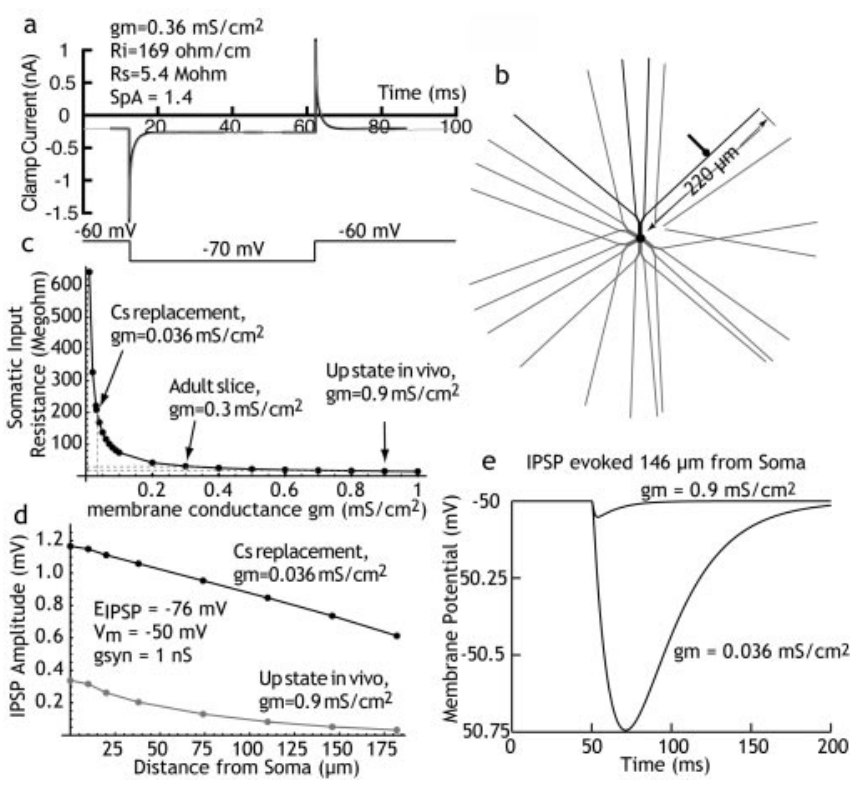

Figure 5. Computer simulation of IPSC generation on striatal spiny neurons. $a$, Voltageclamp results applying a $-10 \mathrm{mV}$ pulse to a spiny neuron recorded using $\mathrm{Cs}^{+}$replacement of $\mathrm{K}^{+}$and loading with $\mathrm{Cl}^{-}$as used in postsynaptic neurons in paired recordings. The gray line is the clamp current; the black line is a best fit for model parameters given in the figures. $b$, Morphological model of the spiny neuron used in the curve fit and the simulations. Dendritic tips are $220 \mu \mathrm{m}$ from the soma, and a single synapse was placed on a single dendrite (black) at various distances from the soma. c, The relationship between somatic input resistance and membrane conductivity for the model neuron with other parameters as determined in the curve fit. The value of $g_{\mathrm{m}}$ associated with the configuration used for voltage-clamped postsynaptic neurons is shown, as is the value estimated from typical measurements of the input resistance of the spiny neuron in the up state in vivo (Wilson and Kawaguchi, 1996). d, Amplitude of simulated IPSCs for synaptic conductances with decay time constant of $12.5 \mathrm{msec}$ (as measured in the experiments), and amplitudes of $1 \mathrm{nS}$, in a spiny neuron at a membrane potential of -50 $\mathrm{mV}$. The effect of the change in membrane conductance on IPSC amplitude is apparent at all of the places along the dendrite but gets increasingly pronounced on the distal dendrites. $V_{m}$, Membrane voltage; $g_{\text {syn }}$, synaptic conductivity. $e$, A comparison of IPSC amplitudes expected for identical IPSCs evoked by dendritic synapses using the $C s^{+}$replacement value of membrane conductance and the value expected from in vivo recording. Note that the IPSC in the in vivo case is $<0.05 \mathrm{mV}$ in amplitude. Increasing the membrane conductance to the range seen in slice recordings $(30-70 \mathrm{M} \Omega$ ) produced IPSCs of $\sim 0.1 \mathrm{mV}$, as reported in current-clamp papers cited (data not shown).

$\mathrm{mV}$, this method yielded input resistance values of 150-200 $\Omega / \mathrm{cm}$ and $\mathrm{SpA}$ values of $1.4-2.0$ [the presence of spines causing membrane area to be increased by $40-100 \%$, which is close to the anatomical measurements of Wilson (1992) and physiological measurements by Reyes et al. (1998) ]. Adjustment of $g_{\mathrm{m}}$ could produce changes in somatic input resistance over a wide range (Fig. 5c). The low input resistance condition was assumed to be identical to the $\mathrm{Cs}^{+}$replacement, except for an increase in $g_{\mathrm{m}}$, and $g_{\mathrm{m}}$ was adjusted to generate a whole-cell input resistance in the range seen in vivo (Sugimori et al., 1978; Wilson and Kawaguchi, 1996; Tepper et al., 1998), or in adult spiny neurons in slices (Nisenbaum and Wilson, 1995). A comparison of IPSP amplitudes evoked by a $1 \mathrm{nS}$ synaptic conductance placed at various distances from the soma is shown in Figure $5 d$. These were calculated using a reversal potential of $-76 \mathrm{mV}$, and are shown for the IPSP as seen from the soma at $-50 \mathrm{mV}$.

\section{Discussion}

Each striatal spiny neuron receives $\sim 10,000$ excitatory inputs from nearly the same number of different afferent neurons (Zheng and Wilson, 2002), but receives less than one-fifth this number of inhibitory inputs arising from local GABAergic interneurons and from the recurrent collaterals of the other spiny cells (for review, see Wilson, 2004). Striatal interneurons serve a strictly feedforward function, receiving excitation from the same cortical and thalamic sources as the spiny cells. They are at least 50-100 times fewer in number than the spiny projection cells (Kawaguchi, 1993; Luk and Sadikot, 2001; Rymar et al., 2004). Each spiny neuron receives inhibitory input from between 4 and 27 FS interneurons, and overlapping sets of interneurons are shared by nearby spiny cells (Koos and Tepper, 1999).

In contrast, all of the spiny neurons contribute inhibitory collaterals to the inhibitory innervation of the other cells in their vicinity. We and others (Czubayko and Plenz, 2002; Taverna et al., 2004) have shown that, in acute slices, each spiny neuron makes synaptic contacts with approximately one-sixth of the neurons within range of its axonal arborization. The axons (and dendrites) of spiny neurons extend for $\sim 200 \mu \mathrm{m}$, and within the volume occupied by one cell in the rat, there are $\sim 2800$ other spiny neurons (Kincaid et al., 1998). If each spiny cell contacts $15 \%$ of those, the number of other neurons contacted is $\sim 420$, and each should receive input from the same number of other spiny cells. The total number of Sp synapses on a spiny cell is probably also much larger than the number of FS synapses. Guzman et al. (2003) have estimated that Sp synapses account for approximately two-thirds of all of the symmetrical synapses on the spiny neurons, with the remaining third divided among dopaminergic inputs and interneuron synapses (including the FS synapse). If each release site represents a synapse, each spiny neuron makes on average two or three synapses with each additional cell it contacts. This makes $840-1260$ synapses total, which agrees with the estimate by Guzman et al. (2003) (but note that their estimate was based on an average of five synapses per cell). This emphasizes the enormous difference in divergence and convergence between the FS cells and the spiny cells, and thus between feedforward and feedback inhibition. There are a lot of Sp synapses, and it takes many more of them to exert an effective influence at the soma, because the IPSPs from the less numerous FS cells are individually many times larger at the soma than those from the spiny cells.

At the level of the synaptic conductance change evoked by an individual quantum, the two synapses are the same. Both show conductance changes of $0.5-1 \mathrm{nS}$ in response to single quanta, corresponding to the opening of $15-30 \mathrm{GABA}_{\mathrm{A}}$ receptor channels. Both also show relatively high release probabilities (at low activation frequencies). This is so although the $S p \rightarrow S p$ synapses often fail, and failures are rare at the FS $\rightarrow$ Sp synapse (Koos and Tepper, 1999, 2002). The difference between the synapses is the number of release sites. The number of release sites for unitary $\mathrm{Sp} \rightarrow$ Sp synapses is approximately three, whereas FS $\rightarrow$ Sp synapses have six or more.

Also contributing to the amplitude difference at the soma is the electrotonic location on the spiny neuron. It has long been known that spiny neurons make most ( $\sim 90 \%$ ) of their synapses on the spiny portion of the dendrites of other spiny cells, among the excitatory inputs (Wilson and Groves, 1980). The FS cells, however, mostly terminate on spiny cell somata (Kita et al., 1990; Kita, 1993; Bennett and Bolam, 1994). The dendrites of the spiny neurons are only $\sim 200 \mu \mathrm{m}$ in length, but they possess potassium channels that cause them to be electrotonically long (Wilson, 1995; Reyes et al., 1998), so they produce a large attenuation of dendritic synaptic potentials.

Mutual inhibition among spiny neurons has sometimes been postulated to impose a competition among spiny neurons (Wick- 
ens et al., 1995; Beiser and Houk, 1998; Plenz and Kitai, 2000; Bar-Gad and Bergman, 2001). In such an arrangement, a small number of spiny cells fire in response to a shared strong afferent excitation, and they suppress all of the others that are less excited. Our results, combined with those of Tunstall et al. (2002), show that the features of $\mathrm{Sp} \rightarrow \mathrm{Sp}$ inhibition are not suited for strong competitive inhibition. The inhibition exerted by a single neuron is too weak to suppress firing in its neighbors, so one would have to imagine that a group of neurons would have to cooperate to suppress activity in one or more other groups. As pointed out by Tunstall et al. (2002), this would be effective only if spiny neurons formed groups that did not inhibit each other, but that had symmetrical inhibition with other groups of cells. Then there could be winner-take-all competition, not among neurons, but among cooperative groups of cells. However, reciprocally connected spiny cell pairs are extremely rare (Tunstall et al., 2002; Taverna et al., 2004). Winner-take-all inhibition relies on the ability of a winner cell to relieve itself of inhibition by inhibiting its competitors. In the arrangement that apparently holds in the neostriatum, any group of active cells that grew large enough to inhibit its neighbors would inhibit its own members just as much as its competitors. For a network of $n$ neurons within synaptic range of each other, interconnected with probability $p$, the number $(m)$ of internally nonconnected groups of cells of size $k$ is given as follows:

$$
m=\left(\frac{n !}{k !(n-k) !}\right)\left((1-p)^{k-1}\right)^{k}
$$

in which

$$
\left(\frac{n !}{k !(n-k) !}\right)
$$

is the number of combinations of $k$ neurons, and $(1-p)^{k-1}$ is the probability that one neuron makes no synapses on other cells in its group of $k$. For $p=1 / 6$, and $3000>n>200$, the mean size of nonconnected groups is $\sim 15$, and groups containing $>30$ neurons are very rare. On average, a group of 15 nonconnected neurons would make only two to three synapses on each cell of another such group. Thus, the asymmetry of connections prevents formation of powerful mutual inhibition among groups of cells, as well as among individual spiny neurons.

One possible functional explanation for the small amplitude of the $\mathrm{Sp} \rightarrow$ Sp synapse is that its effectiveness is modulated, being small in adult animals but perhaps large when the corticostriatal projection is developing (Plenz and Kitai, 2000; Bar-Gad and Bergman, 2001). We tested this possibility by studying the size of the IPSC over the entire period of corticostriatal development. As suggested by anatomical studies (Tepper et al., 1998), these results yielded no indication of an exuberant period in the development of spiny neuron mutual inhibition, but only a gradual increase in synaptic connectivity. Recurrent inhibition, however weak it may be, is strongest in adult animals. Another possibility, that recurrent inhibition may become large at critical times (e.g., periods of high correlation in input fibers), can also be partly addressed by our results. If such modulation does occur, it is unlikely to rely on changes in release probability. The high resting release probability we observed rules out any large increase (more than $\sim 25 \%$ ) in the size of the solitary IPSC. To be larger, changes in synaptic strength would have to arise from an increase in the postsynaptic response to one quantum, or to an increase in release sites. Another possibility is a change in the effective electro- tonic structure of the postsynaptic cell, but this change would not be specific to these synapses; it would affect excitation as well.

In a random network, convergent weak inhibition will exert a normalizing effect, in which all of the neurons are subjected to an inhibition related to the overall level of activity in the network. Striatal lateral inhibition may serve only this function, but there are other possibilities.

Although small when seen from the soma, the IPSPs generated by $\mathrm{Sp} \rightarrow \mathrm{Sp}$ synapses should be very effective in hyperpolarizing restricted parts of the dendrites near their sites of action. These localized hyperpolarizations could act to overcome the local effects of synaptic depolarization, such as removing $\mathrm{Mg}^{2+}$ block of NMDA channels, and enabling calcium current activation and spike backpropagation (Kerr and Plenz, 2002, 2004; Vergara et al., 2003). Also, the electrotonic length of the dendrites of spiny neurons is regulated by voltage-dependent channels and synaptic inputs, and thus varies with membrane potential and synaptic input (Wilson, 1995; Wilson and Kawaguchi, 1996). Local inhibition may act to control the effective spread of excitatory synaptic inputs along the dendrites of striatal neurons. All of these effects would occur without the presence of any strong hyperpolarization that could be seen from the soma (Plenz, 2003).

In contrast, the IPSP evoked by a single IPSC in a spiny neuron is capable of preventing an action potential in the postsynaptic neuron (Koos and Tepper, 1999). In the high-conductance up state, the short time constant of the spiny neuron means that the IPSC from the FS cell could have a short duration of action, and so could play a role in determining the precise timing of action potentials in the spiny neuron.

\section{References}

Akaike N (1997) Gramicidin perforated patch recording and intracellular chloride activity in excitable cells. Prog Biophys Mol Biol 65:251-264.

Bar-Gad I, Bergman H (2001) Stepping out of the box: information processing in the neural networks of the basal ganglia. Curr Opin Neurobiol 11:689-695.

Beiser DG, Houk JC (1998) Model of cortical-basal ganglionic processing: encoding the serial order of sensory events. J Neurophysiol 79:3168-3188.

Bennett BD, Bolam JP (1994) Synaptic input and output of parvalbuminimmunoreactive neurons in the neostriatum of the rat. Neuroscience 62:707-719.

Bolam JP, Clarke DJ, Smith AD, Somogyi P (1983) A type of aspiny neuron in the rat neostriatum accumulates $\left[{ }^{3} \mathrm{H}\right]$ gamma-aminobutyric acid: combination of Golgi-staining, autoradiography, and electron microscopy. J Comp Neurol 213:121-134.

Clements JD, Silver RA (2000) Unveiling synaptic plasticity: a new graphical and analytical approach. Trends Neurosci 23:105-113.

Czubayko U, Plenz D (2002) Fast synaptic transmission between striatal spiny projection neurons. Proc Natl Acad Sci USA 99:15764-15769.

Foldiak P (1990) Forming sparse representations by local anti-Hebbian learning. Biol Cybern 64:165-170.

Guzman JN, Hernandez A, Galarraga E, Tapia D, Laville A, Vergara R, Aceves J, Bargas J (2003) Dopaminergic modulation of axon collaterals interconnecting spiny neurons of the rat striatum. J Neurosci 23:8931-8940.

Hines ML, Carnevale NT (1997) The NEURON simulation environment. Neural Comput 9:1179-1209.

Jaeger D, Kita H, Wilson CJ (1994) Surround inhibition among projection neurons is weak or nonexistent in the rat neostriatum. J Neurophysiol 72:2555-2558.

Kawaguchi Y (1993) Physiological, morphological, and histochemical characterization of three classes of interneurons in rat neostriatum. J Neurosci 13:4908-4923.

Kawaguchi Y, Wilson CJ, Augood SJ, Emson PC (1995) Striatal interneurones: chemical, physiological and morphological characterization. Trends Neurosci 18:527-535.

Kerr JN, Plenz D (2002) Dendritic calcium encodes striatal neuron output during up-states. J Neurosci 22:1499-1512. 
Kerr JN, Plenz D (2004) Action potential timing determines dendritic calcium during striatal up-states. J Neurosci 24:877-885.

Kilman V, van Rossum MCW, Turrigiano GG (2002) Activity deprivation reduces miniature IPSC amplitude by decreasing the number of postsynaptic $\mathrm{GABA}_{\mathrm{A}}$ receptors clustered at neocortical synapses. J Neurosci 22:1328-1337.

Kincaid AE, Zheng T, Wilson CJ (1998) Connectivity and convergence of single corticostriatal axons. J Neurosci 18:4722-4731.

Kita H (1993) GABAergic circuits of the striatum. In: Chemical signaling in the basal ganglia (Arbuthnott GW, Emson PC, eds), pp 51-72. Amsterdam: Elsevier.

Kita H, Kosaka T, Heizmann CW (1990) Parvalbumin-immunoreactive neurons in the rat neostriatum: a light and electron microscopic study. Brain Res 536:1-15.

Koos T, Tepper JM (1999) Inhibitory control of neostriatal projection neurons by GABAergic interneurons. Nat Neurosci 2:467-472.

Koos T, Tepper JM (2002) Dual cholinergic control of fast-spiking interneurons in the neostriatum. J Neurosci 22:529-535.

Luk KC, Sadikot AF (2001) GABA promotes survival but not proliferation of parvalbumin-immunoreactive interneurons in rodent neostriatum: an in vivo study with stereology. Neuroscience 104:93-103.

Nisenbaum ES, Wilson CJ (1995) Potassium currents responsible for inward and outward rectification in rat neostriatal spiny projection neurons. J Neurosci 15:4449-4463.

Plenz D (2003) When inhibition goes incognito: feedback interaction between spiny projection neurons in striatal function. Trends Neurosci 26:436-443.

Plenz D, Kitai ST (2000) Adaptive classification of cortical input to the striatum by competitive learning. In: Brain dynamics and the striatal complex (Miller R, Wickens JR, eds), pp 165-177. Amsterdam: Harwood.

Reyes A, Galarraga E, Flores-Hernandez J, Tapia D, Bargas J (1998) Passive properties of neostriatal neurons during potassium conductance blockade. Exp Brain Res 120:70-84.

Rymar VV, Sasseville R, Luk KC, Sadikot AF (2004) Neurogenesis and stereological morphometry of calretinin-immunoreactive interneurons of the neostriatum. J Comp Neurol 469:325-339.

Scheuss V, Schneggenburger R, Neher E (2002) Separation of presynaptic and postsynaptic contributions to depression by covariance analysis of successive EPSCs at the calyx of Held synapse. J Neurosci 22:728-739.
Sugimori M, Preston RJ, Kitai ST (1978) Response properties and electrical constants of caudate neurons in the cat. J Neurophysiol 41:1662-1675.

Taverna S, Van Dongen YC, Groenewegen HJ, Pennartz CM (2004) Direct physiological evidence for synaptic connectivity between medium-sized spiny neurons in rat nucleus accumbens in situ. J Neurophysiol 91:1111-1121.

Tepper JM, Sharpe NA, Koos TZ, Trent F (1998) Postnatal development of the rat neostriatum: electrophysiological, light- and electron-microscopic studies. Dev Neurosci 20:125-145.

Tunstall MJ, Oorschot DE, Kean A, Wickens JR (2002) Inhibitory interactions between spiny projection neurons in the rat neostriatum. J Neurophysiol 88:1263-1269.

Vergara R, Rick C, Hernandez-Lopez S, Laville JA, Guzman JN, Galarraga E, Surmeier DJ, Bargas J (2003) Spontaneous voltage oscillations in striatal projection neurons in a rat corticostriatal slice. J Physiol (Lond) 553:169-182.

Wickens JR, Kotter R, Alexander ME (1995) Effects of local connectivity on striatal function: stimulation and analysis of a model. Synapse 20:281-298.

Wilson CJ (1992) Dendritic morphology, inward rectification, and the functional properties of neostriatal neurons. In: Single neuron computation (McKenna T, Davis J, Zornetzer SF, eds), pp 141-171. San Diego: Academic.

Wilson CJ (1995) Dynamic modification of dendritic cable properties and synaptic transmission by voltage-gated potassium channels. J Comput Neurosci 2:91-115.

Wilson CJ (2004) The basal ganglia. In: The synaptic organization of the brain, Ed 5 (Shepherd GM, ed.), pp 361-413. Oxford: Oxford UP.

Wilson CJ, Groves PM (1980) Fine structure and synaptic connections of the common spiny neuron of the rat neostriatum: a study employing intracellular injection of horseradish peroxidase. J Comp Neurol 194:599-615.

Wilson CJ, Kawaguchi Y (1996) The origins of two-state spontaneous membrane potential fluctuations of neostriatal spiny neurons. J Neurosci 16:2397-2410.

Zheng T, Wilson CJ (2002) Corticostriatal combinatorics: the implications of corticostriatal axonal arborizations. J Neurophysiol 87:1007-1017. 